\title{
Can we reach a $70 \%$ level of herd immunity to return to normality?
}

\section{Jong-Koo Lee}

Department of Family Medicine, Seoul National University College of Medicine, Seoul, Korea

Received: April 15, 2021

Accepted: April 20, 2021

Corresponding author: Jong-Koo Lee Department of Family Medicine, Seoul National University College of Medicine, 103 Daehak-ro, Jongno-gu, Seoul 03080, Korea E-mail: docmohw@snu.ac.kr
The global second wave of the coronavirus disease 2019 (COVID-19) pandemic peaked in midJanuary 2021, and the spread of COVID-19 then increased again starting in March, cumulatively causing approximately 136 million cases and about 3 million deaths. This third wave of the pandemic has been continuing to spread throughout Europe and Southwest Asia. During and after the second wave of the pandemic, immunizations in Israel, the United Kingdom (UK), and the United States of America significantly reduced deaths and incident cases [1,2]. In Israel, where $58.9 \%$ of the population received more than 10 million vaccinations using the Pfizer vaccine-allowing the population to reach the level of herd immunity in a specific group-the mortality rate was markedly reduced. On the one hand, this example provides a good model of a vaccination campaign, but on the other hand, imported South African variants have led to reinfections, and there is a cautious outlook regarding the possibility that the number of infections will increase due to fatigue with non-pharmaceutical interventions (NPIs) in the young [3]. In the United States of America, more than 189 million doses have been given, with 2-dose vaccinations covering over 74 million individuals, or $22.3 \%$ of the total population. However, since NPIs have been lifted in some states, cases seem to be on the rise once more. Owing to the incidence of unusual blood clot events, the Janssen COVID-19 vaccine was recently withdrawn for urgent use, but it still may be a successful and effective 1-shot vaccine. In the UK, about $48 \%$ of the population has been vaccinated at least once, and the number of cases and deaths has decreased. However, a recent report described deep vein thrombosis with thrombocytopenia linked to the AstraZeneca (AZ) COVID-19 vaccine. When the intensive care unit (ICU) hospitalization rate was compared between individuals who received the AZ vaccine and a group without vaccination, the $\mathrm{AZ}$ vaccine group showed lesser favorable outcomes than the unvaccinated group with a low rate circulation of coronavirus (2 cases per 10,000); therefore, the AZ vaccine is currently not recommended for those under 30. Still, it seems to be a promising vaccine [4]. Chile has vaccinated 12 million people, and $39 \%$ of the population has been vaccinated more than once, but the incidence of cases and deaths remains high, unlike in other countries. Altogether, the uncertainty in the vaccine supply around the world, the hesitation about vaccination due to severe side effects, and the decrease in vaccine efficacy due to the spread of mutant strains mean that it may be difficult to return to normal daily life this year in USA $[5,6]$.

What countermeasures can be taken by our country (the Republic of Korea) in this situation? First of all, we must rapidly increase the vaccination rate. Out of Korea's 79 million contracts for 
vaccines, approximately 1.57 million are being secured and vaccination is being promoted, resulting in a vaccination rate of $2 \%$ of the population [2]. The high-risk group, consisting of people over 65 years old, was assigned to receive vaccinations first and foremost to reduce mortality and the incidence of severe COVID-19 cases. Although some hypersensitivity reactions have been reported, vaccine hesitancy is low, and vaccines must be imported on a continuous basis to meet the government's target immunization level of 70\%. However, in contrast to what the original plan predicted, vaccine supply might be difficult to come by. As the need for vaccination will increase year after year due to the possibility of COVID-19 becoming an endemic disease, now is a critical time to ramp up CMO vaccine production and to create original domestic vaccines using new technologies.

Secondly, with a broader range of variants occurring in some countries, preparedness is more important than expected. B. 1.1.7 has been reported in 132 countries, B.1.351 in 82, and B.1.128.1 in 51. The B.1.351 and B1.1.28.1 variants increase transmissibility and severity, and decrease neutralizing activity. Reinfections have already been reported [7]. Furthermore, it has been reported that vaccines-especially the AZ vaccine-have limited efficacy in preventing infections with the B.1.1.7 variant. For B.1.351, it has been demonstrated that most of the vaccines, including the Moderna, Pfizer, and AZ vaccines, had a lower neutralizing antibody titer, and the $\mathrm{AZ}$ vaccine had no effect on mild cases, leaving an information gap regarding the effectiveness of the vaccine against asymptomatic infections. It was also reported to have a low neutralizing antibody titer against B1.1.28.1. Before the newer variants in Korea become the main epidemic or natural selection creates another new variant in Korea, the vaccination rate should be rapidly increased, and measures to prevent the transmission of variants should be strengthened by monitoring imported cases and quarantining people suspected to be infected. Of course, as the vaccination rate rises, so does the freedom to travel and the possibility of an influx of variants. Supporting both low- and middle-income countries in increasing vaccination rates can ensure that no country falls behind. The submission of a certification of a negative COVID-19 test by travelers should also prevent variants from being exported.

Thirdly, despite the above-mentioned quarantine and vaccination measures, which are effective in reducing severe cases and deaths, there is a gap in the prevention of asymptomatic infection and transmission, making it necessary to maintain social distance and wear a mask to block transmission. Whether or not herd immunity is achieved through vaccination, it will be difficult to return to the previous normal. Long-term countermeasures must be prepared quickly for the winter. As protection that focuses on the elderly in the winter, certain activities (such as visiting nursing homes, various gatherings, sports facilities, religious gatherings, and dining) will be not permitted for people over the age of 65 [6]. Distance working and education will be promoted more aggressively than in previous years. In addition, because there is a high likelihood that the number of severely ill patients will increase and there will be a shortage of ICU beds and workforce, as seen in 2020, measures to secure stable ICU nurses and beds in preparation for the double burden caused by the seasonal influenza epidemic should be supplemented.

Finally, R\&D is a powerful tool to combat emerging reemerging diseases. People must be encouraged to conduct research, research funds must be raised, and the research environment must be improved. Eventually, COVID-19 control and eradication will be a battle between the development of vaccines, treatments, and diagnostics and stockpiling of supplies. There is a particularly pressing need to establish correlates of protection so that vaccine efficacy results obtained with pre-existing variants can be translated to newly emerging variants because it is impractical and time-consuming to repeat clinical trials with each new variant that may exhibit immune escape [8].

\section{Notes}

\section{Ethics Approval}

Not applicable.

\section{Conflicts of Interest}

The author has no conflicts of interest to declare.

\section{Funding}

None.

\section{References}

1. World Health Organization (WHO). WHO coronavirus (COVID-19) dashboard [Internet]. Geneva: WHO; 2021 [cited 2021 Apr 14]. Available from: https://covid19.who.int.

2. Reuters. COVID-19 vaccination tracker [Internet]. Reuters; 2021 [cited 2021 Apr 14]. Available from: https://graphics.reuters.com/worldcoronavirus-tracker-and-maps/vaccination-rollout-and-access/.

3. Lubell M. South African variant may evade protection from Pfizer vaccine, Israeli study says [Internet]. Reuters; 2021 [cited $2021 \mathrm{Apr}$ 14]. Available from: https://www.reuters.com/article/uk-healthcoronavirus-israel-study-idUSKBN2BXOJX.

4. BBC. The US may never use the AstraZeneca vaccine due to contractual reasons says Dr Anthony Fauci, although he thinks it's "a good vaccine" [Internet]. BBC; 2021 [cited 2021 Apr 14]. Available from: https://twitter. com/BBCr4today/status/1381993457296756742?ref_src = twsrc\%5Egoo gle\%7Ctwcamp\%5Eserp\%7Ctwgr\%5Etweet.

5. BBC. Return to normality won't be a light switch moment, says Fauci [Internet]. BBC; 2021 [cited 2021 Apr 14]. Available from: https://www. bbc.com/news/av/health-56731430.

6. Murray CJ, Piot P. The potential future of the COVID-19 pandemic: 
will SARS-CoV-2 become a recurrent seasonal infection? JAMA 2021;325:1249-50.

7. World Health Organization (WHO). Weekly epidemiological update on COVID-19: 13 April 2021. [Internet]. Geneva: WHO; 2021 [cited 2021 Apr 14]. Available from: https://www.who.int/publications/m/item/ weekly-epidemiological-update-on-covid-19---13-april-2021.
8. Karim SS. Vaccines and SARS-CoV-2 variants: the urgent need for a correlate of protection. Lancet 2021;397:1263-4. 